\title{
Diferentes estratégias para aumentar a produção de biossurfactante de um isolado de
}

\section{Paenibacillus sp. (BR13834)}

\author{
Different strategies to increase the biosurfactant production of a Paenibacillus sp. isolate (BR13834) \\ Diferentes estrategias para aumentar la producción de biosurfactantes de un aislado de Paenibacillus
} Sp. (BR13834)

\section{Resumo}

Os biossurfactantes são metabólitos produzidos por diversos microorganismos, e nos últimos anos estão atraindo um interesse na comunidade científica devido suas vantagens em relação aos surfactantes sintéticos. O presente estudo teve como objetivo incrementar a produção de biossurfactante por Paenibacillus sp., otimizando os fatores de crescimento fonte de carbono, $\mathrm{pH}$, temperatura e tempo de cultivo. Inicialmente foram avaliadas as fontes de carbono glicose, lactose, azeite de oliva, óleo de soja, glicerol e querosene. Em seguida, foram avaliados os fatores $\mathrm{pH}$, Temperatura e Tempo, utilizado um delineamento fatorial para a identificação dos fatores que influenciam o processo de produção de biossurfactante. Por fim, os fatores ambientais temperatura e tempo de cultivo foram avaliados usando delineamento central composto rotacional (DCCR). Em todos os ensaios foi utilizado o isolado BR13834 pertencente ao gênero Paenibacillus. O modelo de previsão empírica desenvolvido foi considerado adequado para descrever a produção de biossurfactante em relação à tensão superficial $\left(\mathrm{R}^{2}=0,755\right)$. O valor mínimo para a tensão superficial foi de $34,6 \mathrm{mN} / \mathrm{m}$, obtido nas condições ideais de $30{ }^{\circ} \mathrm{C}$ e 24 horas de cultivo. Os resultados demonstraram que o DCCR foi adequado para identificar as melhores condições de produção de biossurfactante produzido por Paenibacillus sp.

Palavras-chave: DCCR; Biossurfactante; Paenibacillus sp.

\begin{abstract}
Biosurfactants are metabolites produced by several microorganisms, and in recent years are attracting interest in the scientific community due to their advantages over synthetic surfactants. The present study aimed to increase the production of biosurfactants by Paenibacillus sp., optimizing the growth factors carbon source, $\mathrm{pH}$, temperature, and culture time. Initially, the carbon sources glucose, lactose, olive oil, soybean oil, glycerol, and kerosene were evaluated.
\end{abstract}

\footnotetext{
${ }^{1}$ Embrapa Amapá, Rodovia Juscelino Kubitscheck, km 5, Universidade, CEP 68903-419, Macapá, AP, Brasil.

${ }^{2}$ Embrapa Agroindústria Tropical, Rua Doutora Sara Mesquita, 2270 Pici, CEP 60511-110 Fortaleza, CE, Brasil.
} 
Then, the factors $\mathrm{pH}$, Temperature, and Time were evaluated, using a factorial design to identify the factors that influence the process of biosurfactant production. Finally, the environmental factors temperature and cultivation time were evaluated using central composite rotational design (DCCR). In all trials, the isolate BR13834 belonging to the genus Paenibacillus was used. The empirical prediction model developed was found to be adequate to describe biosurfactant production concerning surface tension $\left(\mathrm{R}^{2}=0.755\right)$. The minimum value for surface tension was 34.6 $\mathrm{mN} / \mathrm{m}$, obtained under the optimal conditions of $30^{\circ} \mathrm{C}$ and 24 hours of cultivation. The results showed that DCCR was suitable to identify the best conditions for the production of biosurfactant produced by Paenibacillus sp.

Keywords: DCCR; Biosurfactant; Paenibacillus sp.

\section{Resumen}

Los biosurfactantes son metabolitos producidos por varios microorganismos, y en los últimos años están despertando el interés de la comunidad científica debido a sus ventajas sobre los surfactantes sintéticos. El presente estudio tuvo como objetivo aumentar la producción de biosurfactante por Paenibacillus sp., optimizando los factores de crecimiento fuente de carbono, $\mathrm{pH}$, temperatura y tiempo de cultivo. Inicialmente, se evaluaron las fuentes de carbono glucosa, lactosa, aceite de oliva, aceite de soja, glicerina y parafina. A continuación, se evaluaron los factores $\mathrm{pH}$, temperatura y tiempo, mediante un diseño factorial para identificar los factores que influyen en el proceso de producción de biosurfactantes. Finalmente, se evaluaron los factores ambientales temperatura y tiempo de cultivo mediante un diseño rotacional compuesto central (DCCR). En todos los ensayos se utilizó el aislado BR13834 perteneciente al género Paenibacillus. El modelo empírico de predicción desarrollado resultó adecuado para describir la producción de biosurfactantes en relación con la tensión superficial $\left(\mathrm{R}^{2}=0,755\right)$. El valor mínimo de la tensión superficial fue de 34,6 $\mathrm{mN} / \mathrm{m}$, obtenido en las condiciones ideales de $30^{\circ} \mathrm{C}$ y 24 horas de cultivo. Los resultados demostraron que el DCCR era adecuado para identificar las mejores condiciones para la producción del biosurfactante producido por Paenibacillus sp.

Palabras clave: DCCR; Biosurfactante; Paenibacillus sp.

\section{Introdução}

Os biossurfactantes são moléculas anfipáticas que agem entre fluidos com diferentes polaridades, aumentam a área de contato com compostos insolúveis, reduzem a tensão superficial e interfacial propiciam melhor mobilidade, biodisponibilidade e biodegradação (Bezerra, Rufino, Luna, \& Sarubbo, 2018; Joy, Butalia, Sharma, \& Rahman, 2017). Suas propriedades permitem ampla aplicação em diversas áreas, entre elas nas indústrias alimentícias (produção de emulsificantes alimentares), farmacêutica

e cosmética (formulação de cremes, medicamentos e agentes antimicrobianos), petroquímica, agrícola (produção de fertilizantes), bem como engenharia civil (tratamento de resíduos e esgoto) e em outros segmentos industriais (Al-Wahaibi et al., 2014; Sakthipriya, Doble, \& Sangwai, 2015; Santos, Rufino, Luna, Santos, \& Sarubbo, 2016).

Uma eficiente produção de biossurfactante a nível industrial, requer vários fatores que acabam influenciando na quantidade e tipo de biossurfactante produzido. O uso de diversos fatores (fatores nutricionais e ambientais) utilizando métodos estatísticos, além da utilização de matérias-primas baratas, com o objetivo de reduzir o custo no processo de produção vem sendo utilizado (Deepika, Nagaraju, \& Bramhachari, 2017). O processo de produção ocorre por fermentação de diversos substratos e microorganismos, ocorrendo assim a síntese de diversos metabólitos para aplicações industriais (Satpute, Płaza, \& Banpurkar, 2017). A síntese desses metabólitos pode ser espontânea ou induzida (presença de indutores), sob variações de condições ambientais ( $\mathrm{pH}$, temperatura, arejamento, agitação, tempo) e nutricionais (diferentes fontes de carbono e nitrogênio), ou quando são mantidas em condições de stress no crescimento celular, como uma baixa concentração de nitrogênio (Bhardwaj, Cameotra, \& Chopra, 2013; He, Liu, Yang, \& Liu, 2016; Sakthipriya et al., 2015; Santos et al., 2016).

A identificação e otimização das condições que afetam a produção de biossurfactantes é importante para o desenvolvimento de um processo competitivo em termos de custos (Mukherjee, Das, \& Sen, 2006; Kiran, Thomas, Selvin, Sabarathnam, \& Lipton, 2010).

A metodologia de superfície de resposta é um dos métodos comumente utilizados no processo de otimização de processos. Essa metodologia utiliza um conjunto de técnicas estatísticas para projetar experimentos, construir modelos e simultaneamente avaliar os efeitos dos fatores, estabelecendo assim as condições ótimas para obtenção do produto de interesse 
(Almeida et al., 2017). Outras técnicas como o fatorial fracionado, delineamento central composto rotacional são frequentemente utilizados para otimizar os parâmetros nos processos da produção de metabólitos antimicrobianos, biossurfactantes e enzimas (Deepak et al., 2008; X. Wang, Huang, Kang, Buchenauer, \& Gao, 2010; Fontes, Amaral, Nele \& Coelho, 2010). No fatorial fracionado, apenas uma fração dos experimentos é executada, reduzindo, assim, a quantidade de experimentos quando comparado ao planejamento fatorial completo (Montgomery, 2017). O delineamento composto central rotacional (DCCR) permite avaliar os efeitos combinados e as interações entre as variáveis que influenciam na obtenção do produto de interesse (Santos et al., 2016).

A composição do meio, como fontes de carbono, fontes de nitrogênio, salinidade e outros fatores de crescimento, influenciam fortemente o crescimento celular e o acúmulo de produtos metabólicos, portanto, a otimização desses parâmetros pode melhorar a eficiência bacteriana (Najafi et al., 2011).

O objetivo deste estudo foi otimizar a produção de biossurfactante pela bactéria Paenibacillus sp., variando em diferentes planejamentos experimentais os fatores nutricionais e ambientais no processo de cultivo.

\section{Metodologia}

\subsection{Microorganismo}

Nós utilizamos o isolado FGV 20 (BR13834) de Paenibacillus proveniente da amostra de solo coletada em ambiente de Várzea na cidade de Ferreira Gomes, Amapá. O isolado foi depositado no Centro de Recursos Biológicos Johanna Döbereiner (Embrapa Agrobiologia) e a sequência do gene 16S rDNA depositada no GenBank NCBI com código de acesso MK156435. O acesso ao patrimônio genético foi registrado, sob o código A49223C, no Sistema Nacional de Gerenciamento do Patrimônio Genético (SISGEN), conforme legislação vigente ( $\left.n^{\circ} 13.123 / 2015\right)$.

\subsection{Preparo do inóculo}

O isolado foi repicado em placas contendo meio Agar Nutriente (1,0 g de extrato de carne, 2,0 g de extrato de levedura, 5,0 g de peptona, $5.0 \mathrm{~g}$ de cloreto de sódio, 15,0 g de Agar bacteriológico e 4,0 mL de agente antifúngico Nistatina por litro de água destilada, $\mathrm{pH}=6.8 \pm 0.2$ ) e incubado a $30^{\circ} \mathrm{C}$ por $24 \mathrm{~h}$. Colônias foram transferidas para tubos contendo caldo nutriente e incubadas a $30{ }^{\circ} \mathrm{C}$ por 72 horas sem agitação. Para a avaliação da produção de biossurfactante utilizou-se $1 \mathrm{~mL}$ do inóculo.

\subsection{Estratégias para aumentar a produção do biossurfactante}

Diferentes estratégias foram utilizadas com o objetivo de aumentar a produção do biossurfactante produzido pela Paenibacillus sp. Inicialmente houve o estudo do efeito de algumas fontes de carbono e em seguida a seleção das melhores condições operacionais para a produção do biossurfactante. Após essa etapa, foi realizada a otimização do processo usando Delineamento Central Composto Rotacional.

A produção do biossurfactante foi realizada em meio caldo nutriente $(1,0 \mathrm{~g}$ de extrato de carne, 2,0 g de extrato de levedura, 5,0 g de peptona bacteriológica, 5,0 g de cloreto de sódio e 4,0 $\mathrm{mL}$ antifúngico Nistatina por litro de água destilada, $\mathrm{pH}=6.8 \pm 0.2)$.

Após os ensaios, o extrato obtido foi centrifugado a $6000 \mathrm{rpm}$ e $10 \mathrm{~min}$ a $4{ }^{\circ} \mathrm{C}$ para obtenção do sobrenadante livre de células e utilizado para avaliar a produção do biossurfactante por meio do teste de emulsificação e redução da tensão superficial do meio. 


\subsubsection{Efeito das fontes de carbono sobre a produção de biossurfactante}

As fontes de carbono utilizadas foram glicose (C1), lactose (C2), azeite (C3), óleo de soja (C4), glicerol (C5) e querosene (C6). O caldo nutriente foi suplementado com 5,0\% (p / v) de cada fonte de carbono. Os testes de produção de biossurfactante foram conduzidos utilizando um balão Erlenmeyer de $125 \mathrm{~mL}$ contendo $100 \mathrm{~mL}$ de meio e as culturas foram incubadas a $30{ }^{\circ} \mathrm{C}$ por 96 horas sem agitação.

\subsubsection{Seleção da melhor condição operacional no processo de produção do biossurfactante}

Após avaliação das fontes de carbono foram avaliadas a concentração da fonte de carbono (1,0\% e 5,0\%), tempo de cultivo (24h e $96 \mathrm{~h})$, temperatura de incubação $\left(25^{\circ} \mathrm{C}\right.$ e $\left.40^{\circ} \mathrm{C}\right)$ e $\mathrm{pH}$ do meio de cultivo $(6,0$ e 9,0$)$ sobre a produção de biossurfactante. Utilizou-se para isso um experimento inteiramente casualizado em arranjo fatorial $2^{4}$, totalizando 16 corridas, em triplicata.

\subsubsection{Otimização da produção de biossurfactante por Paenibacillus sp. (FGV 20)}

A produção do biossurfactante foi conduzida em meio caldo nutriente e querosene como agente indutor na produção. Realizou-se ensaio em Delineamento Central Composto Rotacional (DCCR) para otimização dos fatores temperatura e tempo de incubação. O DCCR foi realizado em cinco níveis $(-1,41,-1,0,0,0,1,0$ e 1,41), totalizando 10 corridas com 4 pontos centrais para estimar o erro experimental. Os parâmetros utilizados foram: temperatura $22,9{ }^{\circ} \mathrm{C} ; 25^{\circ} \mathrm{C} ; 30^{\circ} \mathrm{C} ; 35^{\circ} \mathrm{C}$ e $37^{\circ} \mathrm{C}$ e tempo 18,3h, 20h, 24h, 28h e 29,6h sem agitação. A tensão superficial foi utilizada como variável resposta para a produção de biossurfactante.

\subsection{Avaliação da emulsificação e da tensão superficial}

O teste de emulsificação foi realizado através da adição de $2,0 \mathrm{ml}$ de querosene comercial em um tubo de ensaio contendo 2,0 $\mathrm{ml}$ do sobrenadante livre de células. A mistura foi vigorosamente agitada em vórtex por 2 min (Rodrigues, Moldes, Teixeira, \& Oliveira, 2006). As avaliações foram realizadas a temperatura ambiente e o índice de emulsificação (E24h) calculado pela razão entre a altura da coluna de emulsão após 24 h e a altura total da coluna.

Para avaliar a tensão superficial, utilizou-se um tensiômetro KRUSS (Easydyne), conforme metodologia descrita por Kuyukina et al. (2001) utilizando o anel de Du Noüy (du Noüy, 1925). Antes de cada teste, o anel DU NUOY foi esterilizado usando um queimador de Bunsen e calibrado com água destilada $(\sim 70,4 \pm 0,1 \mathrm{mN} / \mathrm{m})$.

\subsection{Análise estatística}

Os dados obtidos foram analisados por análise de variância unidirecional (ANOVA) e as médias comparadas pelo teste de Tukey a 5\% de significância, usando o software Minitab® 19. A análise dos resultados de otimização foi realizada utilizando o programa STATISTIC versão 10.0 da StatSoft, USA.

\section{Resultados e Discussão}

Os fatores que afetam a produção de biossurfactante têm sido estudados nos últimos anos (Najafi et al., 2011). A escolha dos substratos em termos de valor nutricional é fundamental no processo de crescimento do microrganismo e consequentemente no aumento da produção de biossurfactante (Satpute et al., 2017).

Em nosso trabalho o isolado Paenibacillus sp. foi capaz de produzir biossurfactante utilizando as fontes de carbono hidrofílicas e hidrofóbicas (C1 - Glicose, C2 - Lactose, C3 - Azeite de Oliva, C4 - Óleo de soja, C5 - Glicerol e C6 - 
Querosene). Os valores para o índice de emulsificação e para a tensão superficial nas diferentes fontes de carbono, estão demonstradas na Figura 1.

A fonte querosene (C6) apresentou maior valor para o índice de emulsificação e a melhor redução na tensão superficial $40,49 \pm 2,65 \%$ e $36,47 \pm 0,31 \mathrm{mN} / \mathrm{m}$, respectivamente.

Figura 1. Índice de emulsificação após $24 \mathrm{~h}$ e Tensão superficial observados para o sobrenadante livre de células, do cultivo de Paenibacillus sp. FGV 20 (BR 13834).

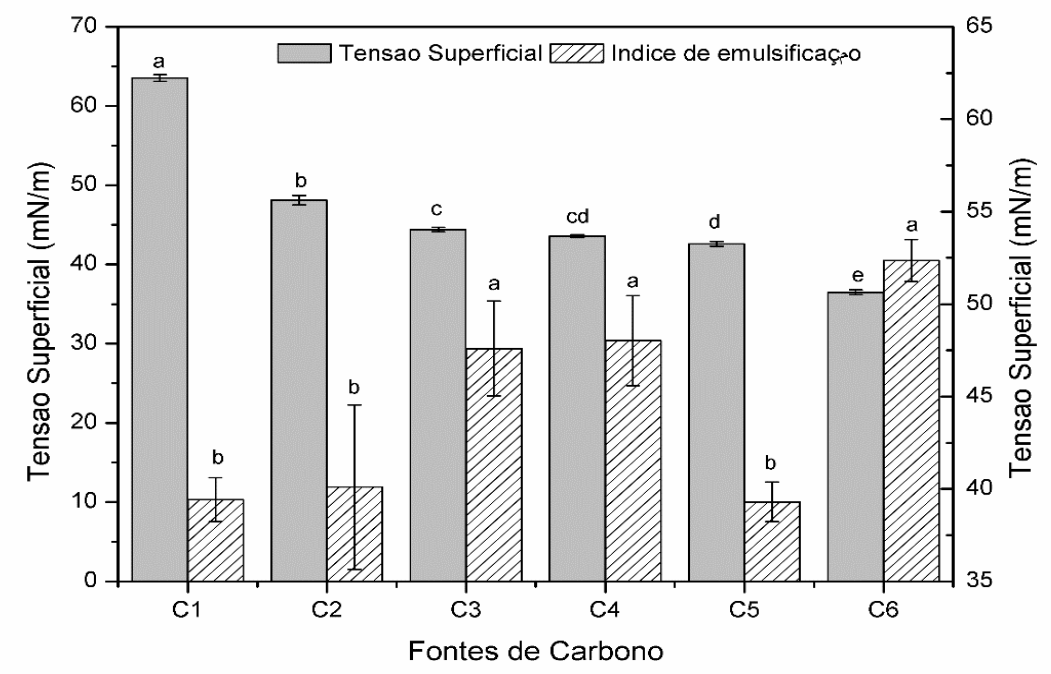

Fonte: Autores (2021).

Substratos hidrofóbicos são reportados por alguns autores como excelentes fontes no processo de produção de biossurfactante. Em trabalho com Paenibacillus lautus e Paenibacillus polymyxa, Omotayo, Egbomeade, Taiwo, Oyebamiji e Ilori (2013) relataram a capacidade desses microrganismos utilizarem petróleo bruto, diesel, querosene, hexadecano e xileno para a síntese de biossurfactante.

Jimoh e Lin (2018) reportaram o n-hexadecano, óleo diesel, n-dodecano e n-tetradecano com bons indutores na produção de biossurfactante utilizando o isolado Paenibacillus sp. D9, com valores de tensão superficial de 28,6 mN/m, 29,4 $\mathrm{mN} / \mathrm{m}, 29,5 \mathrm{mN} / \mathrm{m}$ e $29,7 \mathrm{mN} / \mathrm{m}$, respectivamente a partir do sobrenadante da cultura.

Outros substratos foram reportados por alguns autores. Najafi et al. (2011) relatou valores de tensão superficial inferior a $40 \mathrm{mN} / \mathrm{m}$ a partir da indução com glicose de Paenibacillus alvei ARN63. Em seu trabalho Liang et al. (2013) apresentou resíduos da indústria de processamento de lulas e camarão como fontes de $\mathrm{C} / \mathrm{N}$ no processo de produção de biossurfactante por Paenibacillus macerans TKU029, com valores de redução de tensão superficial de 39,51 e 45,89 mN/m, respectivamente.

Após a avaliação prévia da melhor fonte de carbono, um ensaio em planejamento fatorial foi utilizado para verificar os efeitos significativos no processo de produção do biossurfactante. Na Tabela 1 ficou evidenciado que os efeitos (Fonte de carbono, temperatura e tempo de cultivo) foram significativos na produção de biossurfactante. Foi observado efeito significativo na interação dupla (Temperatura e Tempo) e interação tripla (pH, Temperatura e Tempo). 
Tabela 1. Análise de variância (ANOVA) para a variável dependente Tensão Superficial no processo de seleção dos principais fatores usando fatorial completo.

\begin{tabular}{|c|c|c|c|c|c|}
\hline Fatores & SS & df & MS & $\mathrm{F}$ & $\mathrm{p}$ \\
\hline (1) Fonte de Carbono & 9,6100 & 1 & 9,6100 & 240,25 & 0,041015 \\
\hline (2) $\mathrm{pH}$ & 0,0025 & 1 & 0,0025 & 0,06 & 0,844042 \\
\hline (3) Temperatura & 506,2500 & 1 & 506,2500 & 12656,25 & 0,005659 \\
\hline (4) Tempo & 14,8225 & 1 & 14,8225 & 370,56 & 0,033041 \\
\hline 1 by 2 & 2,7225 & 1 & 2,7225 & 68,06 & 0,076791 \\
\hline 1 by 3 & 4,8400 & 1 & 4,8400 & 121,00 & 0,057716 \\
\hline 1 by 4 & 0,4225 & 1 & 0,4225 & 10,56 & 0,190030 \\
\hline 2 by 3 & 0,1225 & 1 & 0,1225 & 3,06 & 0,330499 \\
\hline 2 by 4 & 1,4400 & 1 & 1,4400 & 36,00 & 0,105137 \\
\hline 3 by 4 & 17,2225 & 1 & 17,2225 & 430,56 & 0,030657 \\
\hline $1 * 2 * 3$ & 1,5625 & 1 & 1,5625 & 39,06 & 0,101003 \\
\hline $1 * 2 * 4$ & 0,6400 & 1 & 0,6400 & 16,00 & 0,155958 \\
\hline $1 * 3 * 4$ & 2,1025 & 1 & 2,1025 & 52,56 & 0,087259 \\
\hline $2 * 3 * 4$ & 8,4100 & 1 & 8,4100 & 210,25 & 0,043835 \\
\hline Error & 0,0400 & 1 & 0,0400 & & \\
\hline Total SS & 570,2100 & 15 & & & \\
\hline
\end{tabular}

$\mathrm{R}^{2}=0,99993 ; \mathrm{R}^{2} \mathrm{Adj}=0,99895$. Fonte: Autores (2021).

O diagrama de Pareto (Figura 2) apresentou os efeitos padronizados significativos para cada fator e suas interações. Segundo Garrido-López e Tena (2005), o comprimento das barras é proporcional ao valor absoluto dos efeitos estimados. A linha vermelha representa $95 \%$ do intervalo de confiança. Os efeitos que cruzam essa linha são valores significativos em relação à resposta. 
Figura 2. Diagrama de Pareto do processo de pré-seleção dos fatores ambientais e nutricional no processo de produção de biossurfactante pela Paenibacillus sp. FGV 20 (BR 13834).

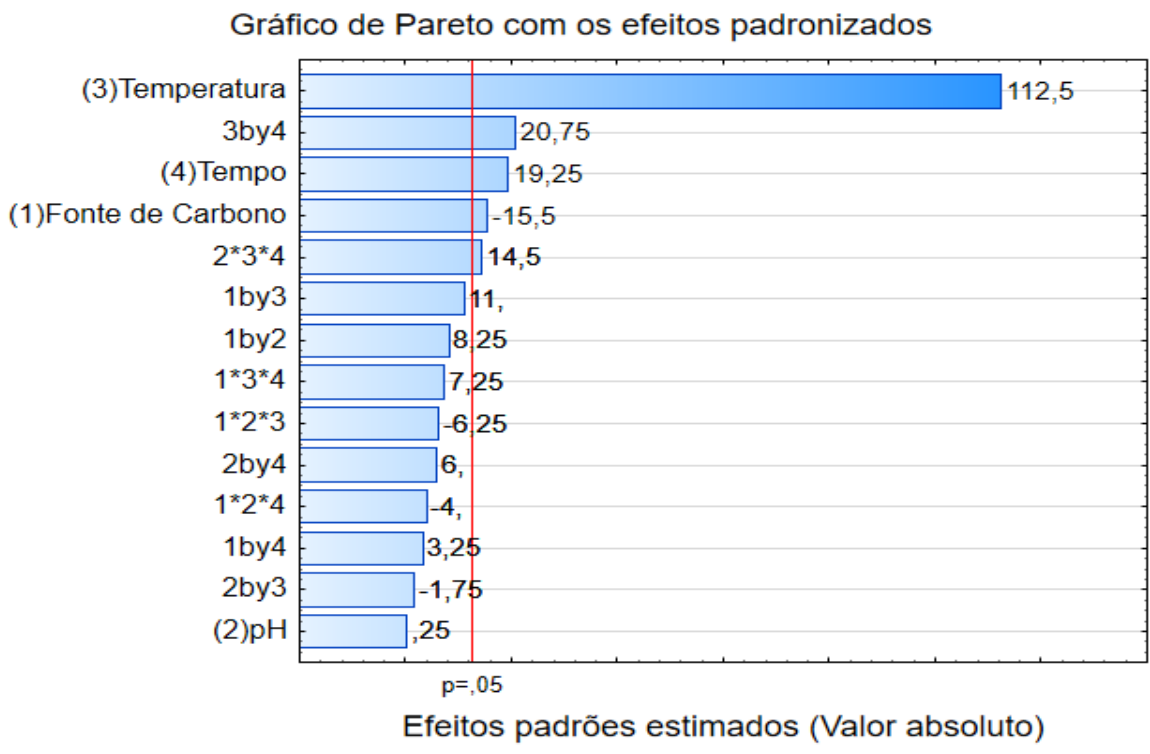

Fonte: Autores (2021).

De acordo com Wang, Huang, Liang, Fang e Wang (2011) a temperatura de incubação foi o fator determinante para a biossíntese de exopolissacarídeos de Paenibacillus sp. TKU023, com temperatura ótima de produção em $37{ }^{\circ} \mathrm{C}$. A temperatura pode favorecer o decréscimo ou aumento da síntese de biossurfactante, pois o aumento da biomassa favorece a liberação dos produtos metabólicos da célula microbiana (Sahoo \& Das, 2011). No presente estudo foram pré-selecionados dois fatores com efeito positivo significativo temperatura e tempo, em seguida foi aplicada a metodologia de Superfície de resposta em delineamento central composto rotacional para otimizar o processo de produção do biossurfactante.

A análise de variância (ANOVA) dos resultados obtidos no processo de otimização da produção de biossurfactante por Paenibacillus sp. FGV 20 (BR13834) não mostrou efeito significativo conforme Tabela 2.

Tabela 2. Análise de variância (ANOVA) da variável dependente Tensão Superficial na otimização do processo de produção.

\begin{tabular}{lccccc}
\hline \multicolumn{1}{c}{ Fatores } & SQ & FV & QM & F & p \\
\hline (1)Temperatura(L) & 15,97646 & 1 & 15,97646 & 39,44805 & 0,100516 \\
\hline Temperatura(Q) & 31,65018 & 1 & 31,65018 & 78,14859 & 0,071710 \\
\hline (2)Tempo (L) & 0,02794 & 1 & 0,02794 & 0,06899 & 0,836478 \\
\hline Tempo (Q) & 5,59446 & 1 & 5,59446 & 13,81349 & 0,167326 \\
\hline 1L by 2L & 5,76000 & 1 & 5,76000 & 14,22222 & 0,165012 \\
\hline Falta de ajuste & 16,86185 & 3 & 5,62062 & 13,87807 & 0,194233 \\
\hline Erro puro & 0,40500 & 1 & 0,40500 & - & - \\
\hline Total SS & 70,68400 & 9 & - & - \\
\hline
\end{tabular}

$\mathrm{R}^{2}=0,75 ; \mathrm{R}_{\text {Adj }}^{2}=0,45$ 
Na Tabela 3 estão apresentadas as variáveis independentes codificadas e normais com os valores da tensão superficial observados e preditos pela equação no processo de otimização da produção de biossurfactante.

Tabela 3. Variáveis codificadas e normais para a otimização por DCCR (Temperatura e tempo) e Tensão superficial do extrato livre de células produzido pela Paenibacillus sp. FGV 20 (BR 13834).

\begin{tabular}{|c|c|c|c|c|c|c|c|}
\hline \multirow{3}{*}{ Corridas } & \multicolumn{2}{|c|}{ Temperatura $\left({ }^{\circ} \mathrm{C}\right)$} & \multicolumn{2}{|c|}{ Tempo (h) } & \multicolumn{3}{|c|}{ Tensão superficial (mN/m) } \\
\hline & Variáveis & Variáveis & Variáveis & Variáveis & Valores & Valores & Dód \\
\hline & Codificada & Normais & Codificada & Normais & observados & Preditos & 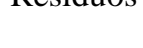 \\
\hline 1 & -1 & 25,0 & -1 & 20,0 & $42,4 \pm 0,19$ & 41,46 & 0,94023 \\
\hline 2 & -1 & 25,0 & +1 & 28,0 & $40,4 \pm 0,33$ & 38,94 & 1,45843 \\
\hline 3 & +1 & 35,0 & -1 & 20,0 & $37,6 \pm 0,38$ & 36,23 & 1,36657 \\
\hline 4 & +1 & 35,0 & +1 & 28,0 & $40,4 \pm 0,42$ & 38,52 & 1,88477 \\
\hline 5 & $-1,41$ & 22,93 & 0 & 24,0 & $41,2 \pm 0,15$ & 42,31 & $-1,11103$ \\
\hline 6 & $+1,41$ & 37,07 & 0 & 24,0 & $36,6 \pm 0,31$ & 38,31 & $-1,71397$ \\
\hline 7 & 0 & 30,0 & $-1,41$ & 18,34 & $36,3 \pm 0,38$ & 37,35 & $-1,04608$ \\
\hline 8 & 0 & 30,0 & $+1,41$ & 29,65 & $35,4 \pm 0,25$ & 37,18 & $-1,77892$ \\
\hline $9(\mathrm{C})$ & 0 & 30,0 & 0 & 24,0 & $35,5 \pm 0,35$ & 35,05 & 0,45000 \\
\hline $10(\mathrm{C})$ & 0 & 30,0 & 0 & 24,0 & $34,6 \pm 0,41$ & 35,05 & $-0,45000$ \\
\hline
\end{tabular}

Fonte: Autores (2021).

No presente estudo nós avaliamos os efeitos da temperatura e do tempo de incubação em DCCR e analisamos por meio de superfície de resposta (Tabela 2 e Figura 3). Foi possível ajustar equação para descrever a produção de biossurfactante (Eq $1)$.

Figura 3. Superfície de resposta que mostra as influências mútuas dos níveis da temperatura e tempo na tensão superficial do meio a partir da produção de biossurfactante por Paenibacillus sp. FGV 20 (BR 13834).
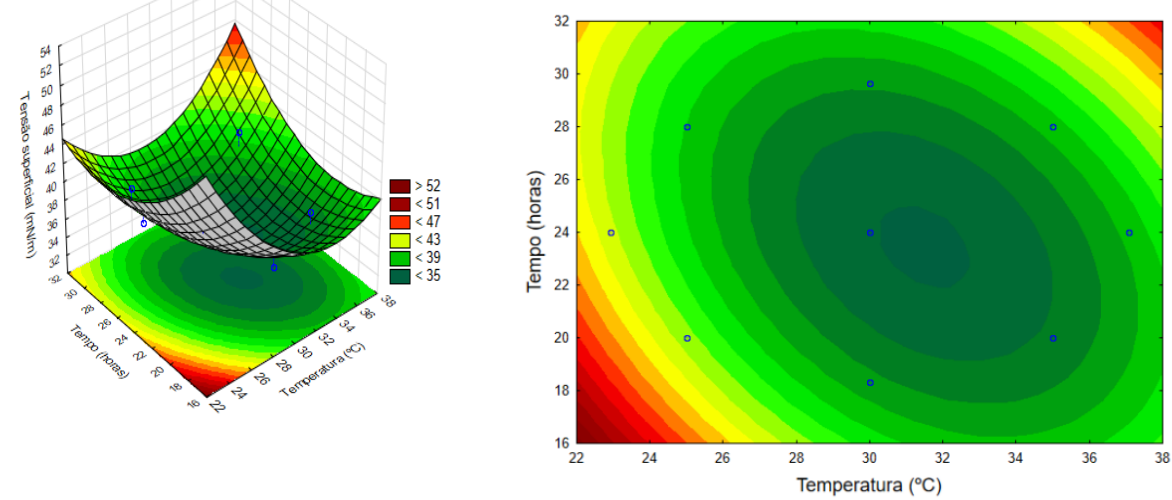

Fonte: Autores (2021). 
$\mathrm{Na}$ Tabela 2 pode ser observado que a falta de ajuste do modelo não foi significativa, indicando que o processo de otimização atingiu os pontos críticos para os regressores que foram escolhidos no modelo (Temperatura e tempo), e com valor para o coeficiente de determinação $\left(R^{2}\right)$ igual a 0,755 . O valor do coeficiente poderia ser elevado pela a adição de outros regressores (fatores), tais como: agitação, concentração de indutor, $\mathrm{pH}$ e etc., o que elevaria o número de experimentos. Devido à natureza complexa dos processos biológicos, conforme Najafi et al. (2011) é muito difícil prever distintamente os efeitos de todos os parâmetros, que podem ter múltiplas interações, essa complexidade pode ter corroborado para o valor encontrado do coeficiente de determinação no processo de otimização da produção de biossurfactante produzido pela Paenibacillus sp.

Dessa forma, o modelo quadrático proposto na Equação 1 é adequado para prever a produção de biossurfactante usando as condições experimentais estabelecidas.

$$
\begin{aligned}
& \text { Tensão superficial }=221,63-8,04 \mathrm{X}_{1}+0,105 \mathrm{X}_{1}^{2}-5,13 \mathrm{X}_{2}+0,0691 \mathrm{X}_{2}^{2}+0,06 \mathrm{X}_{1} \mathrm{X}_{2} \\
& \text { Tensão superficial }=221,63-8,04 \mathrm{X}_{1}+0,105 \mathrm{X}_{1}^{2}-5,13 \mathrm{X}_{2}+0,0691 \mathrm{X}_{2}^{2}+0,06 \mathrm{X}_{1} \mathrm{X}_{2} \text { Eq. (1) }
\end{aligned}
$$

Onde:

$\mathrm{X}_{1}$ - Temperatura; $\mathrm{X}_{2} \mathrm{X}_{2}-$ Tempo;

De acordo com Najafi et al. (2011) a produção de biossurfactante depende da temperatura e do $\mathrm{pH}$, ocorrendo uma produção ótima em uma faixa específica em que a cepa bacteriana é mais ativa, por exemplo mesófilos a 35-40 ${ }^{\circ} \mathrm{C}$ e pH 7 a 7,5. De acordo com o mesmo autor a temperatura ideal de cada tipo de bactéria ocorre em uma faixa relativamente bem definida nas quais esses microorganismos operam com mais eficiência, indicando o caráter dependente da temperatura nos processos de produção de biossurfactante.

Em nosso trabalho as tensões superficiais mais baixas foram observadas na faixa de temperatura entre 25 e $30^{\circ} \mathrm{C}$ e pH 6,0. Trabalhando com Paenibacillus sp. D9, Jimoh e Lin (2018) também encontrou os valores mais baixos para tensão superficial na temperatura de $30^{\circ} \mathrm{C}$. No processo de otimização da produção de biossurfactante por uma cepa de Paenibacillus alvei ARN63 ficou evidenciado que a cepa apresentou faixa ótima pH entre 6 e 8, sendo o maior rendimento no pH de 6,89 (Najafi et al., 2011).

Em relação ao tempo de fermentação, o valor mais baixo da tensão superficial foi observado 24 horas após a inoculação. Na literatura diversos tempos foram relatados durante o processo de otimização, tempos similares (Najafi et al., 2010, 2011) e superiores (Almeida et al., 2017; Amirabadi et al., 2013; Jimoh \& Lin, 2018) ao encontrado em nosso estudo.

\section{Conclusão}

A fonte de carbono querosene foi a que apresentou o melhor índice de emulsificação e melhor redução da tensão superficial.

Temperatura e tempo de cultivo apresentaram efeitos positivos e significativos sobre a tensão superficial.

Foi possível ajustar modelo matemático para descrever o processo de produção de biossurfactante em relação à tensão superficial $\left(\mathrm{R}^{2}=0,755\right)$, com as variáveis tempo e temperatura, indicando o modelo para a produção de biossurfactante produzido pela Paenibacillus sp. FGV20 (BR13834) nas condições ambientais estabelecidas.

Em continuidade serão realizados estudos relacionados com o processo de extração e caracterização por técnicas não destrutivas dos tipos de biossurfactantes metabolizados pelo isolado, bem como a avaliação do potencial de aplicação dos extratos como estabilizantes, antimicrobianos, controle biológico e na biorremediação de ambientes contaminados. 


\section{Agradecimentos}

A Universidade do Estado do Amapá (UEAP), a Embrapa Amapá, a Embrapa Agrobiologia, a Universidade Federal do Amapá (UNIFAP) e ao Instituto Federal de Educação, Ciência e Tecnologia do Amapá (IFAP) por terem disponibilizado a infraestrutura de seus laboratórios para a execução das atividades.

\section{Referências}

Al-Wahaibi, Y., Joshi, S., Al-Bahry, S., Elshafie, A., Al-Bemani, A., \& Shibulal, B. (2014). Biosurfactant production by Bacillus subtilis B30 and its application in enhancing oil recovery. Colloids and Surfaces B: Biointerfaces, 114, 324-333. doi: 10.1016/j.colsurfb.2013.09.022

Almeida, D. G., da Silva, R. de C. F. S., Luna, J. M., Rufino, R. D., Santos, V. A., \& Sarubbo, L. A. (2017). Response surface methodology for optimizing the production of biosurfactant by Candida tropicalis on industrial waste substrates. Frontiers in Microbiology, 8, 1-13. doi: 10.3389/fmicb.2017.00157

Amirabadi, S. S., Jahanmiri, A., Rahimpour, M. R., Nia, B. R., Darvishi, P., \& Niazi, A. (2013). Investigation of Paenibacillus alvei ARN63 ability for biodemulsifier production: Medium optimization to break heavy crude oil emulsion. Colloids and Surfaces B: Biointerfaces, 109, 244-252. doi: 10.1016/j.colsurfb.2013.03.029

Bezerra, K. G. O., Rufino, R. D., Luna, J. M., \& Sarubbo, L. A. (2018). Saponins and microbial biosurfactants: Potential raw materials for the formulation of cosmetics. Biotechnology Progress, 34(6), 1482-1493. doi: 10.1002/btpr.2682

Bhardwaj, G., Cameotra, S. S., \& Chopra, H. (2013). Biosurfactants from Fungi: A Review. Journal of Petroleum \& Environmental Biotechnology, 04(06). doi: $10.4172 / 2157-7463.1000160$

Deepak, V., Kalishwaralal, K., Ramkumarpandian, S., Babu, S. V., Senthilkumar, S. R., \& Sangiliyandi, G. (2008). Optimization of media composition for Nattokinase production by Bacillus subtilis using response surface methodology. Bioresource Technology, 99(17), 8170-8174. doi: 10.1016/j.biortech.2008.03.018

Deepika, K. V., Nagaraju, G. P., \& Bramhachari, P. V. (2017). Optimization of cultural conditions for marine microbial biosurfactant production: Future prospects from untapped marine resources. In M. M. Naik \& S. K. Dubey (Eds.), Marine Pollution and Microbial Remediation, 105-128. doi: 10.1007/978-98110-1044-6_7

du Noüy, P. L. (1925). An interfacial tensiometer for universal use. The Journal of General Physiology, 7(5), 625-632. doi: 10.1085/jgp.7.5.625

Fontes, G. C., Amaral, P. F. F., Nele, M., \& Coelho, M. A. Z. (2010). Factorial design to optimize biosurfactant production by yarrowia lipolytica. Journal of Biomedicine and Biotechnology, 2010, 1-8. doi: 10.1155/2010/821306

Garrido-López, Á., \& Tena, M. T. (2005). Experimental design approach for the optimisation of pressurised fluid extraction of additives from polyethylene films. Journal of Chromatography A, 1099(1-2), 75-83. doi: 10.1016/j.chroma.2005.09.005

He, Z., Liu, G., Yang, X., \& Liu, W. (2016). A novel surfactant, N,N-diethyl-N'-cyclohexylthiourea: Synthesis, flotation and adsorption on chalcopyrite. Journal of Industrial and Engineering Chemistry, 37, 107-114. doi: 10.1016/j.jiec.2016.03.013Jimoh, A. A., \& Lin, J. (2018). Enhancement of Paenibacillus sp. D9 Lipopeptide biosurfactant production through the optimization of medium composition and its application for biodegradation of hydrophobic pollutants. Applied Biochemistry and Biotechnology, 187(3), 724-743. doi: 10.1007/s12010-018-2847-7

Joy, S., Butalia, T., Sharma, S., \& Rahman, P. K. S. M. (2017). Biosurfactant producing bacteria from hydrocarbon contaminted environment. Chemical Engineering Journal, 317, 232-241. doi: 10.1016/j.cej.2017.02.054

Kiran, G. S., Thomas, T. A., Selvin, J., Sabarathnam, B., \& Lipton, A. P. (2010). Optimization and characterization of a new lipopeptide biosurfactant produced by marine Brevibacterium aureum MSA13 in solid state culture. Bioresource Technology, 101(7), 2389-2396. doi: 10.1016/j.biortech.2009.11.023

Kuyukina, M. S., Ivshina, I. B., Philp, J. C., Christofi, N., Dunbar, S. A., \& Ritchkova, M. I. (2001). Recovery of Rhodococcus biosurfactants using methyl tertiary-butyl ether extraction. Journal of Microbiological Methods, 46(2), 149-156. doi: 10.1016/S0167-7012(01)00259-7

Liang, T. W., Wu, C. C., Cheng, W. T., Chen, Y. C., Wang, C. L., Wang, I. L., \& Wang, S. L. (2013). Exopolysaccharides and antimicrobial biosurfactants produced by paenibacillus macerans TKU029. Applied Biochemistry and Biotechnology, 172(2), 933-950. doi: 10.1007/s12010-013-0568-5

Montgomery, D. C. (2017). Design and analysis of experiments eighth edition. In Design (9th ed., Vol. 2). https://doi.org/10.1198/tech.2006.s372

Mukherjee, S., Das, P., \& Sen, R. (2006). Towards commercial production of microbial surfactants. Trends in Biotechnology, 24(11), 509-515. doi: 10.1016/j.tibtech.2006.09.005

Najafi, A. R., Rahimpour, M. R., Jahanmiri, A. H., Roostaazad, R., Arabian, D., \& Ghobadi, Z. (2010). Enhancing biosurfactant production from an indigenous strain of Bacillus mycoides by optimizing the growth conditions using a response surface methodology. Chemical Engineering Journal, 163(3), 188-194. doi: 10.1016/j.cej.2010.06.044

Najafi, A. R., Rahimpour, M. R., Jahanmiri, A. H., Roostaazad, R., Arabian, D., Soleimani, M., \& Jamshidnejad, Z. (2011). Interactive optimization of biosurfactant production by Paenibacillus alvei ARN63 isolated from an Iranian oil well. Colloids and Surfaces B: Biointerfaces, 82(1), 33-39. doi: 10.1016/j.colsurfb.2010.08.010

Omotayo, A. E., Egbomeade, L. O., Taiwo, O., Oyebamiji, O. O., \& Ilori, M. O. (2013). Hydrocarbon degradation by free-living nitrogen-fixing bacteria. Journal of Scientific Research and Development, 14, 75-84. 
Research, Society and Development, v. 10, n. 17, e44101724232, 2021

(CC BY 4.0) | ISSN 2525-3409 | DOI: http://dx.doi.org/10.33448/rsd-v10i17.24232

Rodrigues, L., Moldes, A., Teixeira, J., \& Oliveira, R. (2006). Kinetic study of fermentative biosurfactant production by Lactobacillus strains. Biochemical Engineering Journal, 28(2), 109-116. doi: 10.1016/j.bej.2005.06.001

Sahoo, P., \& Das, S. K. (2011). Tribology of electroless nickel coatings - A review. Materials and Design, 32(4), 1760-1775. doi: 10.1016/j.matdes.2010.11.013

Sakthipriya, N., Doble, M., \& Sangwai, J. S. (2015). Biosurfactant from Pseudomonas species with waxes as carbon source - Their production, modeling and properties. Journal of Industrial and Engineering Chemistry, 31, 100-111. doi: 10.1016/j.jiec.2015.06.013

Santos, D. K. F., Rufino, R. D., Luna, J. M., Santos, V. A., \& Sarubbo, L. A. (2016). Biosurfactants: Multifunctional biomolecules of the 21st century. International Journal of Molecular Sciences, 17(3), 1-31. doi: 10.3390/ijms17030401

Santos, L. F. M. dos., Coutte, F., Ravallec, R., Dhulster, P., Tournier-Couturier, L., \& Jacques, P. (2016). An improvement of surfactin production by B. subtilis BBG131 using design of experiments in microbioreactors and continuous process in bubbleless membrane bioreactor. Bioresource Technology, 218, 944-952 doi: 10.1016/j.biortech.2016.07.053

Satpute, S. K., Płaza, G. A., \& Banpurkar, A. G. (2017). Biosurfactants' production from renewable natural resources: Example of innovativeand smart technology in circular bioeconomy. Management Systems in Production Engineering, 25(1), 46-54. doi: 10.1515/mspe-2017-0007

Wang, X., Huang, L., Kang, Z., Buchenauer, H., \& Gao, X. (2010). Optimization of the fermentation process of actinomycete strain Hhs.015(T). Journal of Biomedicine and Biotechnology, 2010, 1-10. doi: 10.1155/2010/141876

Wang, C. L., Huang, T. H., Liang, T. W., Fang, C. Y., \& Wang, S. L. (2011). Production and characterization of exopolysaccharides and antioxidant from Paenibacillus sp. TKU023. New Biotechnology, 28(6), 559-565. doi: 10.1016/j.nbt.2011.03.003 